\title{
EXCHANGE COUPLING IN GdM COMPOUNDS
}

\author{
K.H.J. Buschow ${ }^{a}$, G.E. Grechnev ${ }^{b}$, A. HJelm ${ }^{c}$, Y. Kasamatsu ${ }^{d}$, \\ A.S. PANFILOV ${ }^{b}$ and I.V. SVEChKaReV $b$ \\ ${ }^{a}$ Van der Waals-Zeeman Institute, University of Amsterdam \\ Valckenierstraat 65, 1018 XE Amsterdam, The Netherlands \\ ${ }^{b} \mathrm{~B}$. Verkin Institute for Low Temperature Physics, Kharkov, 310164, Ukraine \\ ${ }^{c}$ Helax AB, Box 1704, 75147 Uppsala, Sweden \\ ${ }^{d}$ Kure National College of Technology, 2-2-11 Agaminami, Kure 737, Japan
}

The magnetic susceptibility of ferromagnetic $\mathrm{GdM}$ alloys $\left(\mathrm{M}=\mathrm{Cu}_{1-x} \mathrm{Ga}_{x}\right.$, $\mathrm{Mg}, \mathrm{Zn}$ ) has been investigated under helium gas pressure for temperatures above $T_{\mathrm{C}}$. The evaluated pressure derivatives of the paramagnetic Curie temperature, $\mathrm{d} \ln \Theta / \mathrm{d} P$, appeared to be remarkably different for isovalent $\mathrm{GdMg}$ and $\mathrm{GdZn}$ compounds ( -11.2 and $-0.1 \mathrm{Mbar}^{-1}$, respectively). An analysis of the obtained $\mathrm{d} \ln \theta / \mathrm{d} P$ values for $\mathrm{GdCu}_{1-x} \mathrm{Ga}_{x}$ alloys and results of $a b$ initio electronic structure calculations have revealed the essential role of $5 d$ electrons as the mediators of exchange coupling in ferromagnetic GdM compounds. The pressure derivatives of $T_{\mathrm{C}}$ were calculated by employing the modern mean-field theory, as well as the spin-fluctuation model. As a result, good agreement is found with the experimental values of $\mathrm{d} \ln \theta / \mathrm{d} P$.

PACS numbers: 75.10.Lp, 75.20.En

Experimental and theoretical investigations of magnetic properties have been carried out for GdMg and GdZn compounds with $\mathrm{CsCl}$ type structure, and the pseudobinary solid solutions $\mathrm{GdCu}_{1-x} \mathrm{Ga}_{x}$. The magnetic susceptibility of these alloys has been studied under helium gas pressure up to $2 \mathrm{kbar}$ in the temperature range $150 \mathrm{~K}$ (or $T_{\mathrm{C}}$ ) $<T<300 \mathrm{~K}$. The measurements were made by the Faraday method, using a pendulum magnetometer placed into the pressure cell [1]. The relative errors did not exceed $0.05 \%$. The magnetic susceptibility of all compounds investigated obeys the Curie-Weiss law: $\chi=C /(T-\Theta)$, where the Curie constant $C$ is close to the value of $7.89 \mathrm{~K} \mathrm{e.m.u./mole} \mathrm{appropriate} \mathrm{to} \mathrm{the} \mathrm{magnetic} \mathrm{moment}$ of a free $\mathrm{Gd}^{3+}$ ion, and $\Theta$ is the paramagnetic Curie temperature. For all alloys the experimental $d \ln \chi / \mathrm{d} P$ vs. $\chi$ plots do not show any effect of pressure on the Curie constant within the error bars. The values of the evaluated paramagnetic Curie temperatures and their pressure derivatives, $\mathrm{d} \ln \Theta / \mathrm{d} P$, are listed in Table. The derivatives appeared to be remarkably different for isovalent $\mathrm{GdMg}, \mathrm{GdZn}$, and $\mathrm{GdCu}_{0.5} \mathrm{Ga}_{0.5}$ compounds $\left(-11.0,-0.1\right.$, and $+2.4 \mathrm{Mbar}^{-1}$, respectively). This differer.ce can be apparently ascribed to distinct features in their band structures. An analysis of the obtained $\mathrm{d} \ln \Theta / \mathrm{d} P$ values for $\mathrm{GdCu}_{1-x} \mathrm{Ga}_{x}$ alloys has given 
the occupation number derivative $\mathrm{d} n / \mathrm{d} \ln V \simeq-0.1$, which appeared to be in close agreement with the corresponding derivative, calculated for $d$-electrons in $\mathrm{GdCu}$ compound [2]. This agreement gives an evidence that in compounds considered the indirect exchange coupling of the $f$-moments is mediated predominantly by itinerant $d$-electron states.

TABLE

Magnetic ordering temperatures and their derivatives in $\mathrm{GdMg}$ and GdZn compounds, evaluated from the experimental data and calculated according to the theoretical models $[6,5,7]$.

\begin{tabular}{ll|c|c}
\hline \hline Compound & & $\mathrm{GdMg}$ & $\mathrm{GdZn}$ \\
\hline Experiment & $\Theta[\mathrm{K}]$ & 116 & 270 \\
& $\mathrm{~d} \ln \Theta / \mathrm{d} P$ & $-11.2 \pm 0.9$ & $-0.1 \pm 0.05$ \\
& $\mathrm{~d} \ln \Theta / \mathrm{d} \ln V$ & 5.7 & 0.05 \\
\hline Theory & $T_{\mathrm{C}}[\mathrm{K}]$ & 950 & 1060 \\
$([6])$ & $\mathrm{d} \ln T_{\mathrm{C}} / \mathrm{d} \ln V$ & 1.8 & -0.7 \\
\hline Theory & $T_{\mathrm{C}}[\mathrm{K}]$ & 150 & 300 \\
$([5])$ & $\mathrm{d} \ln T_{\mathrm{C}} / \mathrm{d} \ln V$ & 8 & 3 \\
\hline Theory & $T_{\mathrm{SF}}[\mathrm{K}]$ & 190 & 490 \\
$([7])$ & $\mathrm{d} \ln T_{\mathrm{S}} \mathrm{F} / \mathrm{d} \ln V$ & 6 & -0.6 \\
& $T_{\mathrm{C}}[\mathrm{K}]$ & 180 & 410 \\
& $\mathrm{~d} \ln T_{\mathrm{C}} / \mathrm{d} \ln V$ & 5.5 & -0.5
\end{tabular}

For the paramagnetic and ferromagnetic phases of $\mathrm{GdMg}$ and $\mathrm{GdZn}$ compounds $a b$ initio band structure calculations were performed using the LMTO-ASA method [3], as well as a full potential FP-LMTO method [4]. The $4 f$-states are treated as spin-polarized open core states with a Iund's rule restriction for the $4 f$ spin, according to Ref. [5]. The band structures were calculated for a number of lattice constant values close to the experimental ones, giving also the bulk modulus used in the evaluation of volume derivatives, $B_{\mathrm{GdZn}}=0.45 \mathrm{Mbar}$ and $B_{\mathrm{GdMg}}=0.51 \mathrm{Mbar}$. The application of a modified RKKY model to some $\mathrm{RM}_{\mathrm{x}}$ compounds has revealed [2] that the role of the exchange interaction parameter appears to be not a decisive one in the pressure effect on $\Theta$, and the electron transfer of $s p$ - to $d$-states seems more important. The present calculations confirmed the significant partial contributions of $5 d$-electrons to the density of states at the Fermi level $N\left(E_{\mathrm{F}}\right)$ in $\mathrm{GdMg}$ and $\mathrm{GdZn}(\cong 70 \%)$, and almost no $s$-electron states at $E_{\mathrm{F}}(\cong 1 \%)$. Therefore the direct application of the RKKY model seems not justified for GdMg and GdZn compounds.

The $5 d$ electrons are by far less localized than $4 f$ electrons, and expected to play a significant role in the indirect exchange coupling, provided the rare earth concentration in the compound is high enough to ensure overlap of the $5 d$ wave functions, and provided the partial $5 d$ contribution to $N\left(E_{\mathrm{F}}\right)$ is also high. The 
fulfillment of these requirements for $\mathrm{GdMg}, \mathrm{GdZn}$, and the pseudobinary alloys with close occupation numbers is apparently confirmed by present experiments and band structure calculations. The related mean-field theory, focused on the contribution of $d$-like electrons to the indirect exchange interaction, was proposed for the magnetic ordering temperature [5]

$$
k_{\mathrm{B}} T_{\mathrm{C}}=\frac{1}{3}\left(g_{J}-1\right)^{2} J(J+1) J_{f d}^{2} \widetilde{S}\left(\chi_{d}-\chi_{i i}\right),
$$

where $J_{f d}$ and $J_{d d}$ are the exchange integrals, $\left(g_{J}-1\right)^{2} J(J+1)$ is the de Gennes factor of $\mathrm{Gd}$. The spin susceptibility of conduction $d$-electrons, $\chi_{d}$, is modified by removing the local or on-site susceptibility $\chi_{i i}$, which is responsible for moment formation; $\widetilde{S}=\left[1-J_{d d}\left(\chi_{d}-\chi_{i i}\right) / 2\right]^{-1}$. It improves a conventional mean-field theory [6], giving a considerable reduction of the Curie temperature, when $\chi_{i i}$ is comparable in magnitude to $\chi_{d}$. We attempted to choose $\chi_{i i}$ values to get a reasonable fit to $T_{\mathrm{C}}$ and their volume derivatives. All other parameters in Eq. (1) were taken from the present band structure calculations. It appears that this theory cannot explain the difference in the experimental data, regarding to $\mathrm{d} \ln T_{\mathrm{C}} / \mathrm{d} \ln V$ in these compounds (see Table), on condition that the volume dependence of $\chi_{i i}$ is negligible.

The Curie temperatures were also evaluated here with the spin-fluctuation theory of Mohn-Wohlfarth [7], which relates the calculated spin-polarized electronic structure to $T_{\mathrm{C}}$. Though the theory [7] was developed for itinerant electron systems, and a successful treatment of rare earths has not been expected on its basis, the $T_{\mathrm{C}}$ behaviour in $\mathrm{R}-\mathrm{Fe}$ compounds has been well described with this model in Ref. [8]. Spin fluctuation effects were recently observed in transport, magnetic and thermal properties of the related $\mathrm{GdAg}$ compound [9]. Therefore it seems justified to employ the model [7] also to GdMg and GdZn. The Curie temperature is given by the following equation:

$$
T_{\mathrm{C}}^{2} / T_{\mathrm{CS}}^{2}+T_{\mathrm{C}} / T_{\mathrm{SF}}-1=0 .
$$

$T_{\mathrm{CS}}$ is the calculated Curie temperature based on the mean-field Stoner theory [6], and $T_{\mathrm{SF}}$ is a characteristic temperature, describing the influence of spin fluctuations and related to details of the ferromagnetic $N(E)$ at the Fermi level: $T_{\mathrm{SF}}=M_{\mathrm{c}}^{2} / 10 k_{\mathrm{B}} \chi_{0} . \mathrm{M}_{\mathrm{c}}$ is the magnetic moment of conduction electrons, and the exclange-enhanced ferromagnetic susccptibility $\chi_{0}$ is given by

$$
\chi_{0}^{-1}=\frac{1}{4 \mu_{\mathrm{B}}^{2}}\left[N\left(E_{\mathrm{F}}\right)_{\uparrow}^{-1}+N\left(E_{\mathrm{F}}\right)_{\downarrow}^{-1}-2 I\right] .
$$

IIere $N\left(E_{\mathrm{F}}\right)_{\uparrow}$ and $N\left(E_{\mathrm{F}}\right)_{\downarrow}$ are the spin-polarized contributions to the ferromagnetic $N(E), I$ is the Stoner integral. All these quantities are determined from the calculated ferromagnetic electronic structures at zero-temperature. The pronounced competition is found between "spin-up" and "spin-down" contributions of $d$-electrons to $N(E)$ in GdMg. The steeply sloping $N_{d}(E)$ curves run across each other in the vicinity of $E_{\mathrm{F}}$, and are especially responsive to the atomic volume changes. On the other laand, in GdZn the corresponding $d$-partial contributions are pushed apart by $3 d-5 d$ hybridization.

According to Eq. (2), the required $T_{\mathrm{C}}$ values appeared to be close to the corresponding $T_{\mathrm{SF}}$ for both compounds considered. As can be seen from the Table, 
the spin-fluctuation model represents the $\mathrm{d} \ln \theta / \mathrm{d} \ln V$ derivatives and the relation between the $T_{\mathrm{C}}$ values for $\mathrm{GdMg}$ and $\mathrm{GdZn}$. The agreement of the results of the Mohn-Wohlfarth theory employed with the experimental data points to significance of spin fluctuations in determining the magnetic properties of heavy rare earth metal compounds RM with ferromagnetic ordering.

This work was supported, in part, by the European program INTAS under grant 94-2903, and the Swedish Royal Academy of Sciences. We thank Dr. M.S.S. Brooks and Dr. J.M. Wills for the computer programs used to calculate the band structures.

\section{References}

[1] A.S. Panfilov, Physics and Technique of High Pressure 2, 61 (1992) (in Russian).

[2] G.E. Grechnev, A.S. Panfilov, I.V. Svechkarev, K.H.J. Buschow, A. Czopnik, J. Alloys Comp. 226, 107 (1995).

[3] H.L. Skriver, The LMTO Method, Springer, Berlin 1984.

[4] J.M. Wills, unpublished; J.M. Wills, B.R. Cooper, Phys. Rev. B 36, 3809 (1987).

[5] M.S.S. Brooks, S. Auluck, T. Gasche, J. Trygg, L. Nordström, L. Severin, B. Johansson, J. Magn. Magn. Mater. 104-107, 1496 (1992).

[6] D. Bloch, D.M. Edwards, M. Shimizu, J. Voiron, J. Phys, F 5, 1217 (1975).

[7] P. Mohn, E.P. Wollfarth, J. Phys. F 17, 2421 (1987).

[8] J.P. Woods, B.M. Patterson, A.S. Fernando, S.S Jaswal, D. Welipitiya, D.J. Sellmyer, Phys. Rev. B 51, 1064 (1995).

[9] M.M. Amado, R.P. Pinto, M.E. Braga, J.B. Sousa, P. Morin, J. Magn. Magn. Mater: 153, 107 (1996). 